Journal of

\section{Synchrotron \\ Radiation}

ISSN 1600-5775

Received 28 June 2013

Accepted 4 December 2013

\title{
Design and performance of a new VIS-VUV photoluminescence beamline at UVSOR-III
}

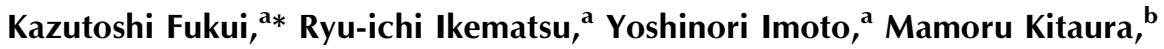 \\ Kazumichi Nakagawa, ${ }^{\mathrm{c}}$ Takao Ejima, ${ }^{\mathrm{d}}$ Eiken Nakamura, ${ }^{\mathrm{e}}$ Masahiro Sakai, \\ Masami Hasumoto ${ }^{\mathrm{e}}$ and Shin-ichi Kimura ${ }^{\mathrm{e}}$
}

\begin{abstract}
${ }^{\mathbf{a}}$ Faculty of Engineering, University of Fukui, 3-9-1 Bunkyo, Fukui 910-8507, Japan, ${ }^{\mathbf{b}}$ Faculty of Science, Yamagata University, 1-4-12 Kojirakawamachi, Yamagata 990-8560, Japan, 'Graduate School of Human Development and Environment, Kobe University, 3-11 Tsurukabuto, Nada-ku, Kobe, Hyogo 657-8501, Japan, d Institute of Multidisciplinary Research for Advanced Materials, Tohoku University, 2-1-1 Katahira, Aoba-ku, Sendai, Miyagi 980-8577, Japan, and ' UVSOR Facility, Institute for Molecular Science, 38 Nishigo-Naka, Myodaiji, Okazaki, Aichi 444-8585, Japan. *E-mail: fukui@fuee.u-fukui.ac.jp
\end{abstract}

\begin{abstract}
A new bending-magnet beamline with a $2.5 \mathrm{~m}$ normal-incidence monochromator has been constructed to serve with a light source in the visible-vacuumultraviolet region for photoluminescence, transmission and reflection spectroscopies of solids at the UVSOR-III $750 \mathrm{MeV}$ synchrotron radiation light source. The aim is to pave the way to establishing a beamline with high photon flux, high brilliance, high energy-resolution, high linear-polarization and low higher-order light. To obtain high photon flux and brilliance, the acceptance angle of the bending-magnet radiation was designed to be $40 \mathrm{mrad}(\mathrm{H}) \times 14 \mathrm{mrad}(\mathrm{V})$ and the post-mirror system employed Kirkpatrick-Baez optics. The incidence angle of the incoming light to the optical elements, except to the gratings, was set to a grazing angle in order to keep a degree of linear polarization. For achieving high energy-resolution, an off-plane Eagle-type monochromator was adopted. Higher-order unwanted light in the energy range below $\sim 11 \mathrm{eV}$ was suppressed to be less than $0.1 \%$.
\end{abstract}

Keywords: beamline; normal-incidence monochromator; photoluminescence.

\section{Introduction}

Solid-state optical devices in the ultraviolet (UV) region have brought about significant improvements in telecommunication, illumination, environmental and medical applications, and are expected to lead to further progress. For technological and scientific research on such industrially important UV materials, optical spectroscopic instruments that cover a wide photon energy region, from the visible (VIS) to the vacuum-ultraviolet (VUV) regions, are required.

We have developed a new bending-magnet beamline, HOTRLU (High-resOlution Transmission, Reflection and LUminescence spectroscopies of solids in the VIS-VUV region), equipped with a $2.5 \mathrm{~m}$ normal-incidence monochromator (NIM) at beamline BL3B of UVSOR-III in order to realise accurate photoluminescence (PL) spectroscopy of such materials in the VIS-VUV regions. HOTRLU has also been constructed as a succession beamline of BL1B (1 m Seya-Namioka-type monochromator beamline), which was closed down at the end of March 2011. HOTRLU is featured as the third specific VIS-VUV beamline at UVSOR-III: a multipurpose beamline ( $3 \mathrm{~m}$ McPherson-type monochromator beamline, BL7B; see Fukui et al., 2001), a variably polarized angle-resolved photoemission beamline with a $10 \mathrm{~m}$ modified Wadsworth-type monochromator (SAMRAI, BL7A; see Kimura et al., 2010), and a new PL-dedicated beamline (HOTRLU). In this report we describe the design details and present performance of the HOTRLU beamline.

\section{Design}

The beamline monochromator for PL measurements is required to satisfy a wide photon energy range, giving a high brilliance on the sample, while maintaining a high degree of linear polarization, a high energy-resolution and high purity (inclusion of less higher-order light). Thus we adopted the following target specifications for the beamline using examples from the performance of the BL7B beamline: a photon energy coverage range of $2-24 \mathrm{eV}$, photon flux $(E / \Delta E$ $=1000)$ higher than $10^{10}$ photons $\mathrm{s}^{-1}$, energy resolution $(E / \Delta E)$ higher than 10000, beam spot size $(\mathrm{H} \times \mathrm{V})$ less than $0.8 \mathrm{~mm} \times$ $0.8 \mathrm{~mm}$, and degree of linear polarization higher than 0.7 .

Since a NIM can maintain both incident beam profile and polarization of the outgoing beam, and the reflectivity is functional up to $\sim 30 \mathrm{eV}$, it is a good solution for achieving the above beamline specifications. Considering the high-resolution specification, we adopt an off-plane Eagle-type NIM (see Namioka, 1959). We use a verticaldispersion grating configuration where both entrance and exit slits are located in the same plane. Even though the reflectivity of the normal incidence is functional up to $\sim 30 \mathrm{eV}$, the square of the reflectivity on the higher energy side becomes low and is not appropriate for PL measurements. Therefore, the layout of this beamline needs to consist of a normal-incidence grating with the other optical elements at grazing incidence. This configuration also preserves the degree of linear polarization of the bending-magnet 
Table 1

Characteristics of optical components of the HOTRLU beamline.

\begin{tabular}{|c|c|c|c|c|c|}
\hline Pre-mirrors & $\begin{array}{l}\text { Incidence } \\
\text { angle }\left(^{\circ}\right)\end{array}$ & \multicolumn{2}{|c|}{ Radius (mm) } & $\begin{array}{l}\text { Dimensions } \\
(\mathrm{mm})\end{array}$ & $\begin{array}{l}\text { Coating } \\
\text { material }\end{array}$ \\
\hline M0 & 85 & \multicolumn{2}{|c|}{$\infty$ (plane) } & $110 \times 300$ & $\mathrm{Au}$ \\
\hline M1 & 81.1 & \multicolumn{2}{|c|}{$773.60 \times 32300$ (toroidal $)$} & $210 \times 280$ & $\mathrm{Au}$ \\
\hline M2 & 87 & \multicolumn{2}{|l|}{$\infty$ (plane) } & $30 \times 440$ & $\mathrm{Au}$ \\
\hline Gratings & $\begin{array}{l}\text { Deviation } \\
\text { angle }\left({ }^{\circ}\right)\end{array}$ & $\begin{array}{l}\text { Radius } \\
(\mathrm{mm})\end{array}$ & $\begin{array}{l}\text { Dimensions } \\
(\mathrm{mm})\end{array}$ & $\begin{array}{l}\text { Coating } \\
\text { material }\end{array}$ & $\begin{array}{l}\text { Grooves } \\
\left(\mathrm{mm}^{-1}\right)\end{array}$ \\
\hline G1 & 4 & 2500 & $40 \times 110$ & $\mathrm{Au}$ & 1200 \\
\hline G2 & 4 & 2500 & $40 \times 110$ & $\mathrm{Pt}$ & 600 \\
\hline G3 & 4 & 2500 & $40 \times 110$ & $\mathrm{Al}$ & 300 \\
\hline
\end{tabular}

\begin{tabular}{llcll}
\hline Post-mirrors & $\begin{array}{l}\text { Incidence } \\
\text { angle }\left({ }^{\circ}\right)\end{array}$ & Radius $(\mathrm{mm})$ & $\begin{array}{l}\text { Dimensions } \\
(\mathrm{mm})\end{array}$ & $\begin{array}{l}\text { Coating } \\
\text { material }\end{array}$ \\
\hline M3 & 82 & 9390 (spherical) & $40 \times 400$ & $\mathrm{Au}$ \\
M4 & 86.5 & 17090 (spherical) & $50 \times 270$ & $\mathrm{Au}$ \\
\hline
\end{tabular}

radiation. However, the floor shape at the beamline usually spreads out toward the end of the beamline. This implies that the layout of the NIM beamline with normal-incidence grating configuration is disadvantageous because user experiment space becomes insufficient. To avoid this, we rotate the monochromator and the post-mirror system in the horizontal plane, and introduce a plane mirror into the pre-mirror system. This mirror also acts as a higher-order cut filter if it is vertically split-coated. The optical layout in the vertical direction is also designed to satisfy the following two conditions: (i) the output beam of the HOTRLU beamline is horizontal, and (ii) the height of the output beam is $\sim 1200 \mathrm{~mm}$. These conditions facilitate PL experiments on condensed matter. For the PL measurements, not only a high photon flux but also high brilliance is required. Thus, for good focusing at the experimental point we employ Kirkpatrick-Baez optics (see Kirkpatrick \& Baez, 1948). An optical layout simulation was carried out using the ray-tracing program SHADOW (see Lai \& Cerrina, 1986).

The adopted parameters of the optical components are listed in Table 1 , and the top and side views are schematically shown in Figs. 1(a) and 1(b), respectively. The acceptance angle of the beamline is $40 \mathrm{mrad}(\mathrm{H}) \times 14 \mathrm{mrad}(\mathrm{V})$, which satisfies the prerequisite photon flux. M0 is a plane mirror for changing the direction of the bending-magnet radiation at the point $\mathrm{S}$ and for reducing heat load. A radiation shield wall is located in between the M0 and M1 mirrors, where a toroidal mirror M1 focuses incoming bending-magnet radiation to the entrance slit $\mathrm{S} 1(1: 1)$ to prevent the focal spot shape from deforming owing to a wide acceptance angle. A plane mirror M2
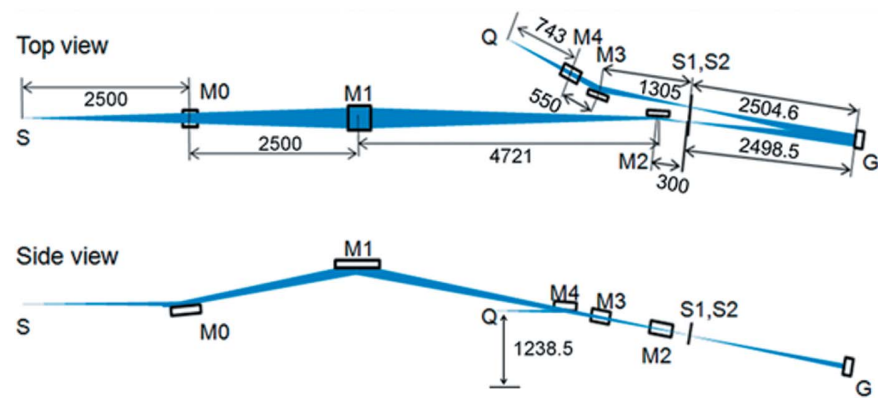

Figure 1

Schematic layout of the HOTRLU. (a) Top view and (b) side view. Dimensions are given in millimeters. reflects the light in the horizontal direction to reserve working space around the sample $(\mathrm{Q})$ and to correct horizontal beam misalignment, as mentioned above. An off-plane Eagle-type monochromator with focal length of $2.5 \mathrm{~m}$ consists of S1, gratings $\mathrm{G}$ and exit slit S2. The monochromator satisfies both $F$-number matching to the pre-mirror system as well as the prerequisite resolution specification. We use three gratings (G1, G2 and G3) with different grooves, blazing angles and coatings to cover the wide photon energy region from 2 to $30 \mathrm{eV}$. The horizontal deviation angle of $\mathrm{G}$ is $4^{\circ}$. The total rotation angle (angle resolution) of the gratings and total linear travel (travel resolution) of the grating chamber were $6^{\circ}\left(0.036^{\circ}\right)$ and $20 \mathrm{~mm}$ $(0.01 \mathrm{~mm})$, respectively. Four optical low-energy-pass filters (LPFs), LiF, quartz, WG32 and OG52, are available and a motorized linear actuator can set one of them just after S2, to reject higher-order light in the low-energy region. The post-mirror system with KirkpatrickBaez optics consists of two spherical mirrors M3 and M4 which condense incoming light from S2 horizontally and vertically. The M4 mirror also realigns the beam parallel to the floor. Finally, the horizontally propagating beam is focused onto the $\mathrm{Q}$ point. Both the VIS-UV and VUV spectrometers with CCD sensors for PL measurements can be placed in the sample chamber. The calculated degree of linear polarization on the paraxial ray of this layout is expected to be higher than 0.7 over the whole energy region.

\section{Present performances}

Fig. 2 shows the throughput spectra measured at the $\mathrm{Q}$ point by a $\mathrm{Si}$ photodiode (SPD; AX-UV100, IRD). The slit widths of S1 and S2 were set to $100 \mu \mathrm{m}$. The photon number estimated using the typical

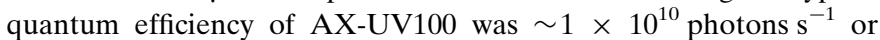
more over the whole photon energy rage from $1.7 \mathrm{eV}$ to $\sim 35 \mathrm{eV}$, at a beam current of $300 \mathrm{~mA}$, which is the typical current of the top-up operation of UVSOR-III. Fig. 3 shows the system throughput with the G3 grating for the above-mentioned LPF variation. The LPF cutoff photon energies are $11.8 \mathrm{eV}(\mathrm{LiF}), 7.7 \mathrm{eV}$ (quartz), $3.9 \mathrm{eV}$ (WG32) and $2.4 \mathrm{eV}$ (OG52). We have confirmed that the intensity of the higher-order light is less than $0.1 \%$ of that of the first-order light; thus we can obtain high-purity monochromated light by using the LPFs in the G3 range below $\sim 11 \mathrm{eV}$. This photon energy range is very important for the PL measurement of solids.

Fig. 4 shows a contour map representing the beam spot profile at the $\mathrm{Q}$ point, obtained by raster scan of a metallic plate with a $0.2 \mathrm{~mm}$ diameter pinhole placed just before the SPD and measuring output current. The profile shown in Fig. 4 was recorded using G2 at $\sim 12 \mathrm{eV}$ $(\sim 100 \mathrm{~nm})$. The full width at half-maximum (FWHM) of the profile

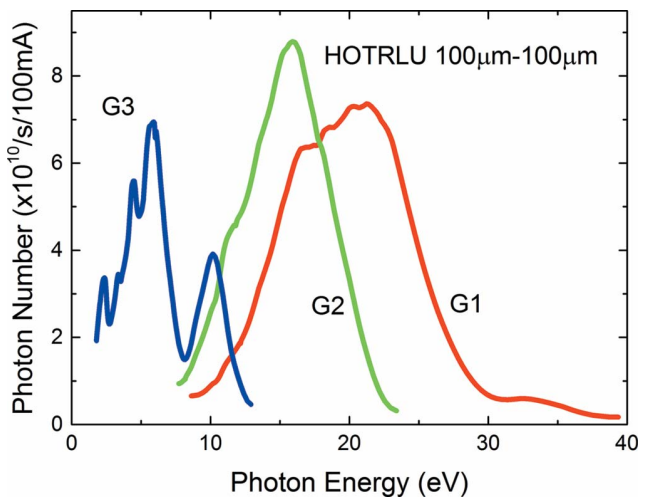

Figure 2

Throughput spectra of the HOTRLU beamline. 


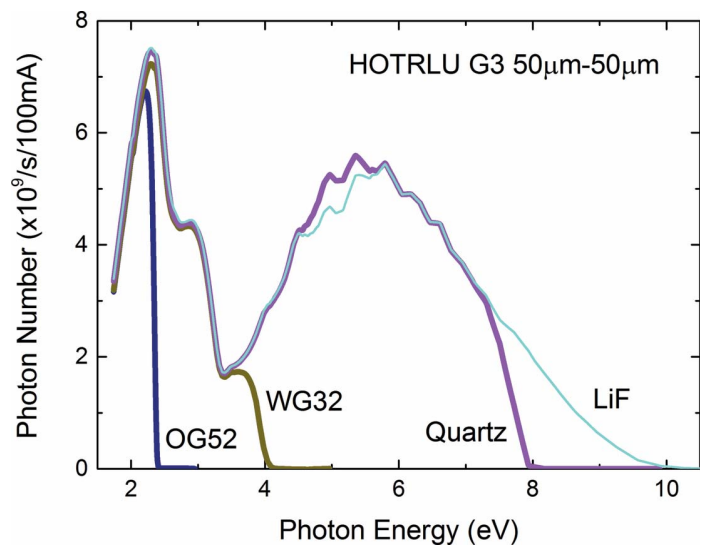

Figure 3

Throughput spectra with the G3 grating described in Table 1, measured with a lowenergy-pass filter.

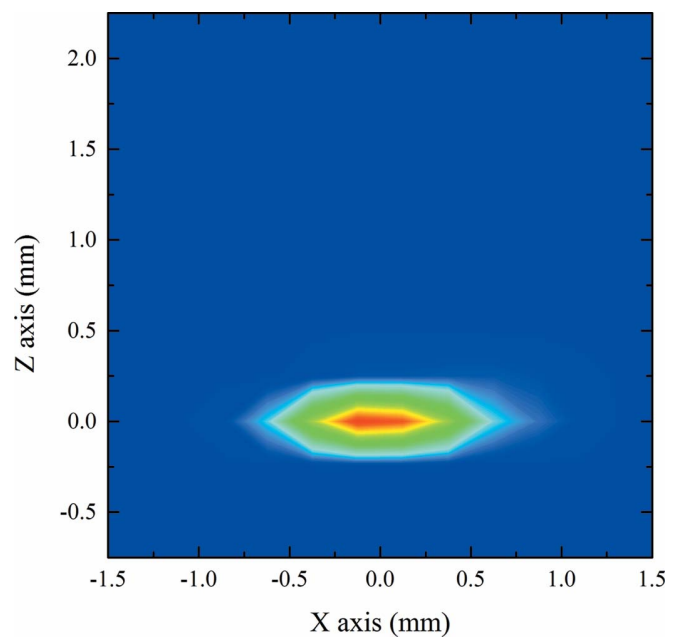

Figure 4

Beam profile contour map image at the sample position measured at $100 \mathrm{~nm}$ using the G2 grating described in Table 1.

was evaluated to be $0.75 \mathrm{~mm}(\mathrm{H}) \times 0.25 \mathrm{~mm}(\mathrm{~V})$. Beam profiles for the other gratings were similar to that of G2 shown in Fig. 4. However, for all gratings, profile deformations were clearly observed at the low photon energy limit.

The absorption spectrum of oxygen molecules in the spectral range of the Schumann-Runge bands, measured with G1 at slit widths S1 and $\mathrm{S} 2$ of $50 \mu \mathrm{m}$, is shown in Fig. 5. Here the horizontal axis, representing wavelength, is not calibrated. In this spectrum the energy (wavelength) resolution $(E / \Delta E)$ for $50 \mu \mathrm{m}$ slit opening is roughly 5000 or more.

In Fig. 6 we show typical PL and PL excitation (PLE) spectra of a $\mathrm{Gd}_{3} \mathrm{Ga}_{3} \mathrm{Al}_{2} \mathrm{O}_{12}$ crystal (GAGG; see Kamada et al., 2012) taken at the HOTRLU beamline. The sample size and the measurement temperature were $10 \mathrm{~mm} \times 5 \mathrm{~mm}$ and $6 \mathrm{~K}$, respectively. The PL spectrum (thick blue line) was measured using a $30 \mathrm{~cm}$ CzernyTurner monochromator with an entrance slit width of $100 \mu \mathrm{m}$ and with a liquid-nitrogen-cooled back-illuminated CCD. The excitation energy was set to $6.52 \mathrm{eV}$ by G3, and both S1 and S2 were set to $300 \mu \mathrm{m}$. A sharp PL peak at $3.92 \mathrm{eV}$ was assigned to the $4 f$ intraatomic emission of $\mathrm{Gd}^{3+}$ ions. The thin black line is a PLE spectrum of the sharp emission peak at $3.92 \mathrm{eV}$, which was measured up to $30 \mathrm{eV}$ by successively replacing the grating among the G3, G2 and G1

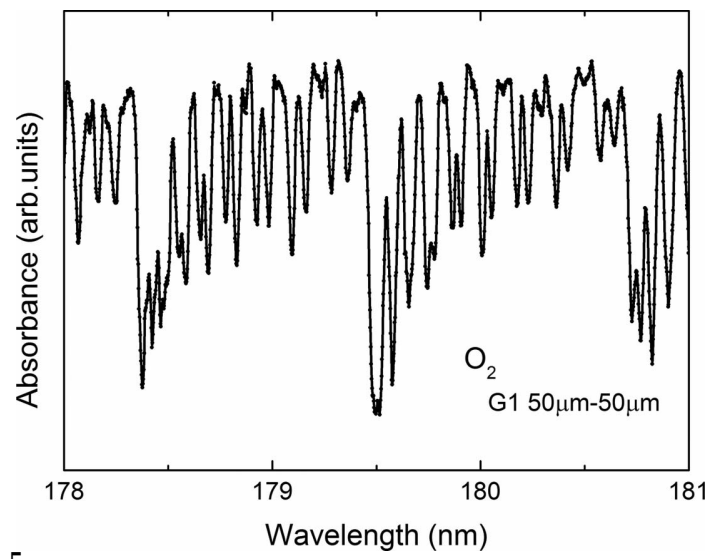

Figure 5

Absorption spectrum in the spectral range of the Schumann-Runge oxygen molecule band.

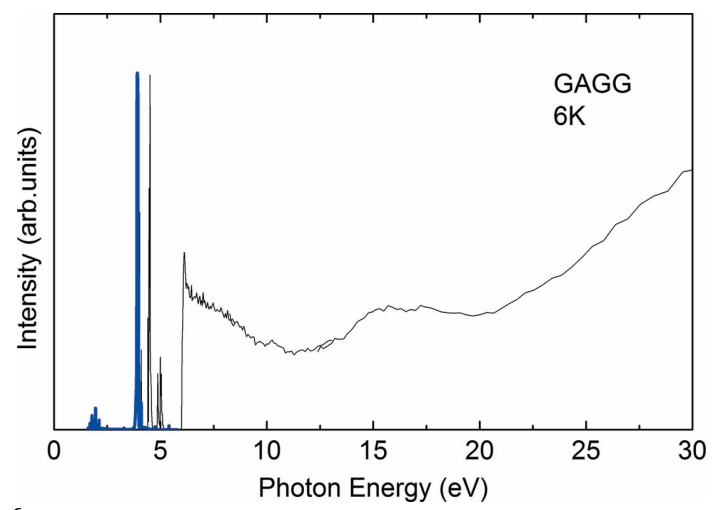

Figure 6

Photoluminescence (thick blue line) and photoluminescence excitation (thin black line) spectra of a $\mathrm{Gd}_{3} \mathrm{Ga}_{3} \mathrm{Al}_{2} \mathrm{O}_{12}(\mathrm{GAGG})$ single crystal measured at $6 \mathrm{~K}$. The excitation energy of the PL spectrum and the emission energy of the PLE spectrum are $6.52 \mathrm{eV}$ and $3.92 \mathrm{eV}$, respectively.

gratings. In the PLE spectrum, sharp peaks owing to $4 f$ intra-atomic absorptions of $\mathrm{Gd}^{3+}$ ions and a clear sharp edge corresponding to the band-to-band absorption of the GAGG are clearly observed at $4-5 \mathrm{eV}$ and at $5.96 \mathrm{eV}$, respectively. A gradual increase of the intensity in the higher energy range $(>20 \mathrm{eV})$ is also observed, which is interpreted as the appearance of multiple electron-hole pairs in GAGG.

\section{Summary}

A new bending-magnet beamline at BL3B has been constructed (HOTRLU) equipped with a $2.5 \mathrm{~m}$ normal-incidence monochromator. The present performance of HOTRLU is as follows (the parameters in square brackets are our target performance at the design stage): photon energy coverage, $1.7-31 \mathrm{eV} \mathrm{[2-24} \mathrm{eV];} \mathrm{photon}$ flux $(E / \Delta E=1000), \geq 10^{10}$ photons s$^{-1}(300 \mathrm{~mA})^{-1}$ over a whole region $\left[\geq 10^{10}\right.$ photons $\left.^{-1}(300 \mathrm{~mA})^{-1}\right]$; energy resolution $(E / \Delta E)$ $(\mathrm{G} 1$, S1 $50 \mu \mathrm{m}, \mathrm{S} 250 \mu \mathrm{m}), \geq 5000[\geq 10000]$; beam spot size at a sample position $(\mathrm{H} \times \mathrm{V}), \sim 0.75 \mathrm{~mm} \times 0.25 \mathrm{~mm}[\leq 0.8 \mathrm{~mm} \times$ $0.8 \mathrm{~mm}]$. Both the polarization evaluation and the resolution improvement are future issues.

We gratefully thank Dr K. Kamada from Materials Laboratory of Furukawa Co. Ltd for providing precious samples. 


\section{beamlines}

\section{References}

Fukui, K., Miura, H., Nakagawa, H., Shimoyama, I., Nakagawa, K., Okamura, H., Nanba, T., Hasumoto, M. \& Kinoshita, T. (2001). Nucl. Instrum. Methods Phys. Res. A, 467-468, 601-604.

Kamada, K., Yanagida, T., Endo, T., Tsutumi, K., Usuki, Y., Nikl, M., Fujimoto, Y., Fukabori, A. \& Yoshikawa, A. (2012). J. Cryst. Growth, 352, 88-90.
Kimura, S., Ito, T., Sakai, M., Nakamura, E., Kondo, N., Horigome, T., Hayashi, K., Hosaka, M., Katoh, M., Goto, T., Ejima, T. \& Soda, K. (2010). Rev. Sci. Instrum. 81, 053104.

Kirkpatrick, P. \& Baez, A. V. (1948). J. Opt. Soc. Am. 38, 766-774.

Lai, B. \& Cerrina, F. (1986). Nucl. Instrum. Methods Phys. Res. A, 246, $337-$ 341.

Namioka, T. (1959). J. Opt. Soc. Am. 49, 460. 